\title{
A DIGITAL MEMS OPTICAL SWITCH
}

\section{Roman C. Gutierrez, Tony K.Tang, Robert Calvet, Darrell Harrington, Stephen Vargo, Indrani Chakraborty*}

Siimpel Corporation, 400 East Live Oak Ave., Arcadia, CA, USA

*Bain \& Company, Inc. 1901 Avenue of the Stars, Los Angeles, CA, USA

\begin{abstract}
This paper summarizes the work done in the development of a commercial three dimensional digital MEMS optical switch and its unique reliability challenges. The paper describes the design, fabrication, and testing of the three-dimensional steering MEMS mirror for the digital optical switch.
\end{abstract}

\section{INTRODUCTION}

Optical switches based on microelectromechanical systems (MEMS) have been the subject of substantial development over the past ten years [1-4]. Due to the requirements for small size and low cost systems, there has been a widely acknowledged need for using digitally controlled mirrors in the optical switch. Digital control eliminates the need for closed loop control in the positioning of each mirror in an optical switch. This is achieved using optical elements made from precise MEMS structures that "snap" into a multitude of predefined positions. This paper presents the design, fabrication and test of a highly reliable digital MEMS mirror with up to 32 positions.

This work was partially funded by the Advanced Technology Program, managed by NIST. We would also like to acknowledge Professor Roger Howe for his support on understanding the reliability issues of these devices.

\section{OPTICAL SWITCH OVERVIEW}

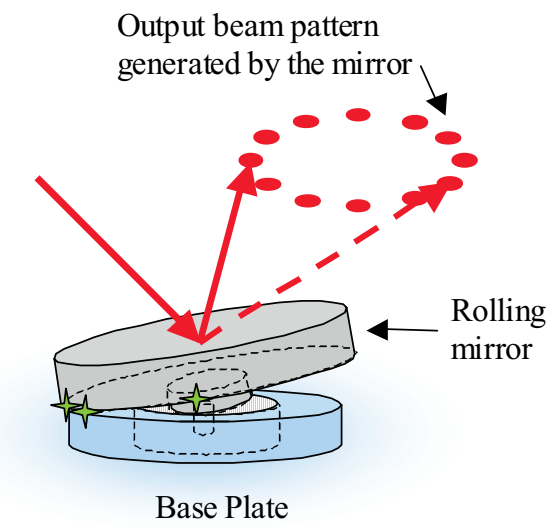

Figure 1. The digital MEMS mirror uses simple mechanical features that allow the mirror to point in precise, predefined positions every time.

Figure 1 illustrates the MEMS mirror of this digital optical switch. This design allows the mirror to "snap-down" to a number of precisely predefined positions. These positions are defined with submicron accuracy using a photolithographic process. In this approach, the mirror is in contact with the baseplate and rolls about a central pivot. The pivot allows the mirror to access $\mathrm{N}$ different snap-down positions distributed in a circle around the central pivot. The plane of the mirror is precisely defined kinematically by three points of contact: one on the central pivot and two on the perimeter of the circle. An electrode is used to electrostatically attract the mirror to this position. A beam of light reflecting from this mirror traces out a cone as the mirror rolls to its $\mathrm{N}$ different positions.

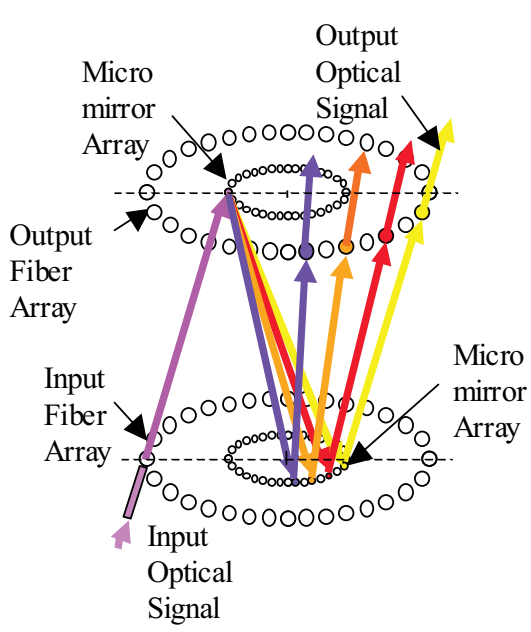

Figure 2. The optical design for a switch based on one-stage digital MEMS mirrors.

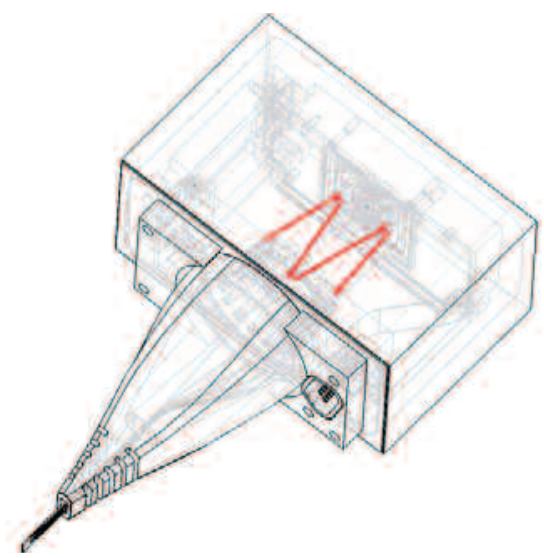

Figure 3. Schematic of an $8 x 8$ digital MEMS optical switch shows the path of light within the switch.

Figure 2 illustrates how an NxN digital MEMS optical switch works using this type of MEMS mirror. The light enters the switch through an optical fiber and is collimated by a lens. The combined fiber and lens is referred to as a collimator. Since the one-stage digital MEMS 
mirror can direct light to points on a circle, the mirrors and fibers are arranged in circles. The mirror array circle is concentric with the fiber array circle. The collimated beams are pointed such that the light hits the mirrors on the opposite side. Each fiber, therefore, has its corresponding mirror. Using this structure, light from any input fiber can be directed to any output fiber with less than $3 \mathrm{~dB}$ of optical loss.

Figure 3 illustrates the layout of an $8 \times 8$ optical switch using this type of digital MEMS mirror. A ribbon fiber with 16 individual single mode fibers is fed into a circular collimator array. Directly opposing this collimator array is a circular array of 16 MEMS mirrors, where each mirror has 8 digitally controlled positions. The red arrows illustrates how the light travels from one collimator to another, by bouncing off a first MEMS mirror, a fixed mirror, and a second MEMS mirror. The $8 \times 8$ optical switch includes all of the drive electronics within the hermetically sealed package.

\section{MEMS MIRROR FABRICATION}

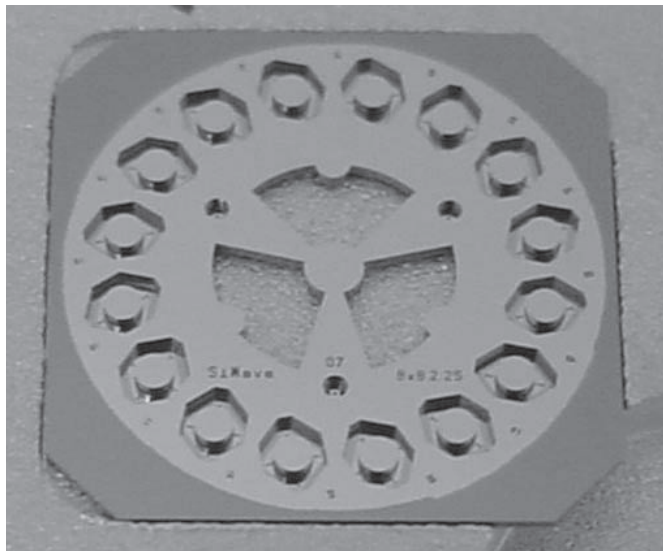

Figure 4. Photographs of the MEMS mirror (left) and baseplate (right) for a digital MEMS optical switch.

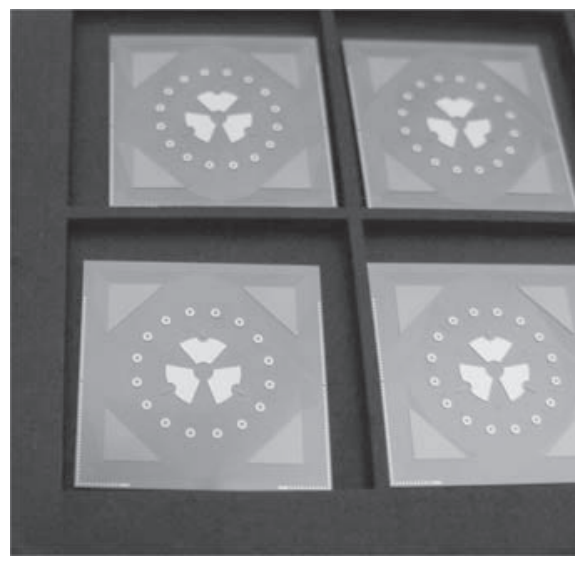

Figure 5. Photographs of the MEMS mirror (left) and baseplate (right) for a digital MEMS optical switch.

The digital MEMS mirrors require three dimensional structures built using inherently planar photolithographic and etch processes. The digital MEMS mirror is composed of two components that are separately fabricated and then assembled together: the MEMS mirror and the baseplate. Figures 5 and 6 show photographs of the MEMS mirror and baseplate structures for the $8 \times 8$ optical switch.

SOI (Silicon on Insulator) wafers are used to make these structures, and the process is outlined in Figure 6. The mirror is made by first patterning and etching the suspension and pivot on the silicon epilayer and then cutting out the mirror on the substrate. Deep Reactive Ion Etching (DRIE) is used to etch both the epilayer and the substrate. The last step consists in depositing a $\mathrm{Ti} / \mathrm{Pt} / \mathrm{Au}$ layer to increase the reflectivity of the mirror in the near infrared.

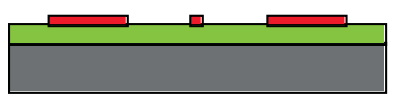

Oxidation

Oxide pattern

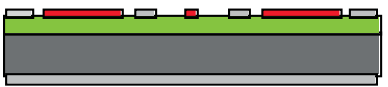

Metallization

Metal pattern

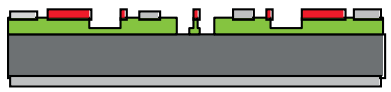

Epilayer pattern

DRIE etch

\section{Baseplate}

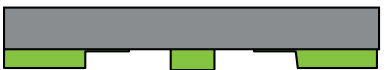

Epilayer pattern

DRIE etch

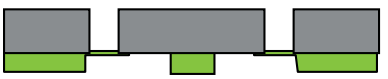

Substrate pattern

DRIE etch

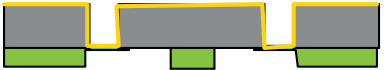

Metallization

\section{Mirror}

Figure 6. Process sequence to fabricate MEMS base-plates and mirrors using single SOI wafers.

The baseplate fabrication consists of a double DRIE etch on the epilayer. This is achieved by using a double patterning of oxide and photoresist, similar to the embedded masking process described by Mita et al. [5]. The metal paterning is used to electrically contact the epilayer, since the silicon is used to carry the electrical signals for electrostatic actuation.

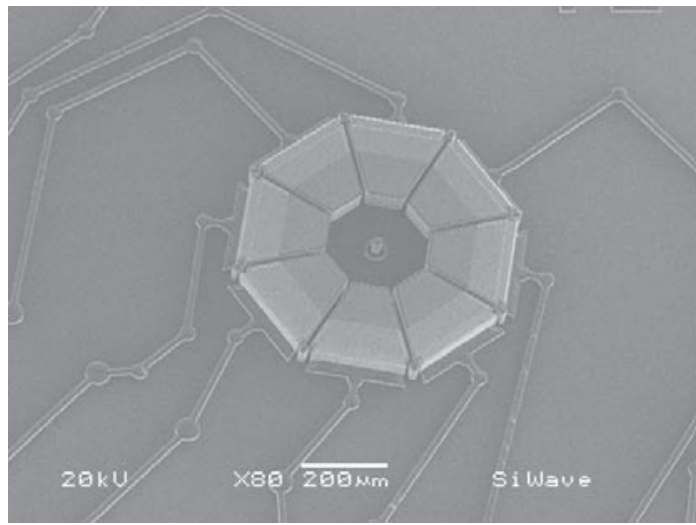

Figure 6. SEM photographs of the MEMS baseplate. 
In order to set the pointing angle of the mirror accurately, all of the kinematic points that set the digital positions of the mirror must be defined in one mask layer. This is done during the first oxidation patterning allowing the highest possible accuracy. Figures 6 and 7 show SEMs of the finished baseplate for one mirror in a $8 \times 8$ switch mirror array. In these pictures, the wedges are the electrodes, and between the electrodes on the outer perimeter are the kinematic points on which the mirror rests when it is actuated. Each electrode has a silicon trace to electrically contact it to a pad that is on the rim of the chip.

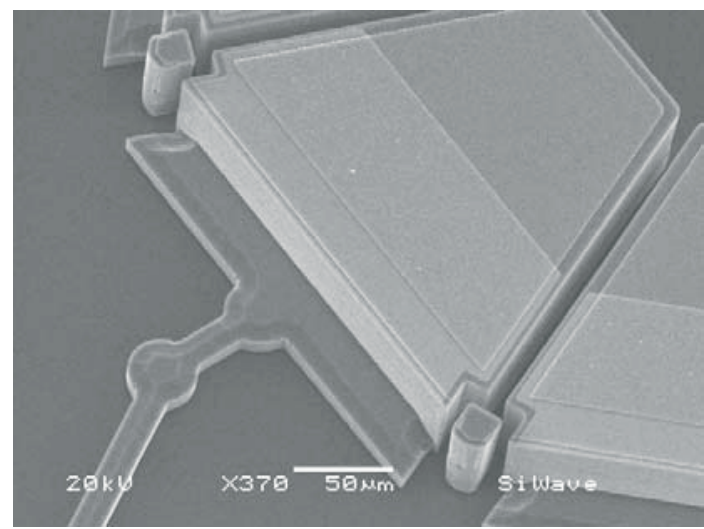

Figure 7. SEM photographs of the MEMS baseplate.

\section{FUNCTIONAL AND RELIABILITY TESTS}

This type of MEMS mirror relies on contacting surfaces, and the reliability of such contact was a significant issue, in contrast to the situation for conventional "macro" machines. A comparison of the surface-to-volume ratio of MEMS and their macroscopic counterparts explains the underlying physical reason for the predominance of surface phenomena in determining MEMS performance. Because surface forces dominate over body forces as the size of a mechanical component is scaled down in size, the control of these forces becomes very important in MEMS design.

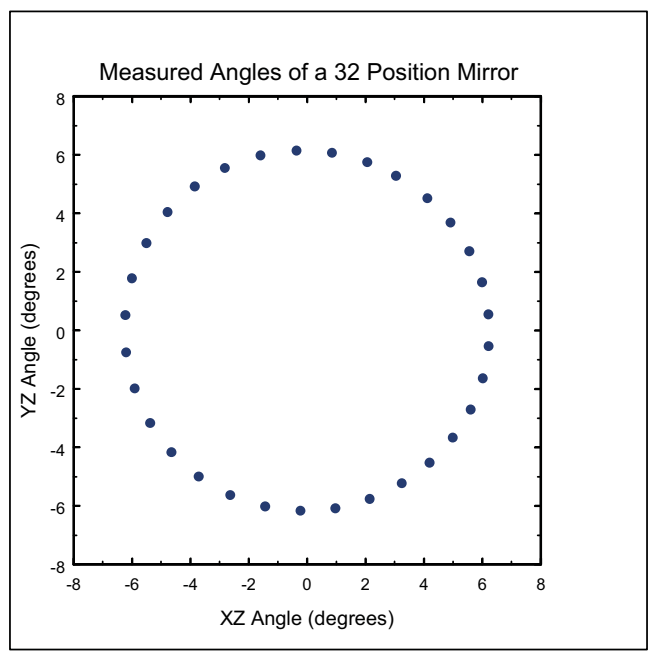

Figure 8. Data of the mirror during 250,000 cycles around all 32 positions. Repeatability in pointing angle is 0.003 degrees.
Previous MEMS research has demonstrated repeatedly that contacting surfaces can easily adhere in the microscale [6-8], a phenomenon called "stiction," and that they may also exhibit very high friction coefficients and be subject to significant wear. The forces involved in contacting surfaces on the micro scale have been the subject of substantial research over the past twenty years. The relative magnitude of surface forces is: capillary (adsorbed water) $>>$ electrostatic $>$ van der Waals. In the case of oxidized silicon surfaces in room air, the work of adhesion (the energy needed to separate contacting surfaces) due to capillary forces can exceed that due to van der Waals attraction by a factor of $10^{4}$. The optimal surface coating for minimizing stiction and wear would be both hydrophobic (eliminating capillary forces) and hard; ideally, contact would occur over as small an area as possible.

This MEMS switch technology has several features that make the problem of contacting surfaces tractable. For example, mirrorbaseplate contact occurs at lithographically defined "kinematic points." The material and contacting edge shape of the kinematic point is engineered to reduce local shear loads and stiction forces. A second important feature is that the basic mirror motion during actuation is rolling, rather than sliding. As a result, wear at the kinematic points is greatly reduced.
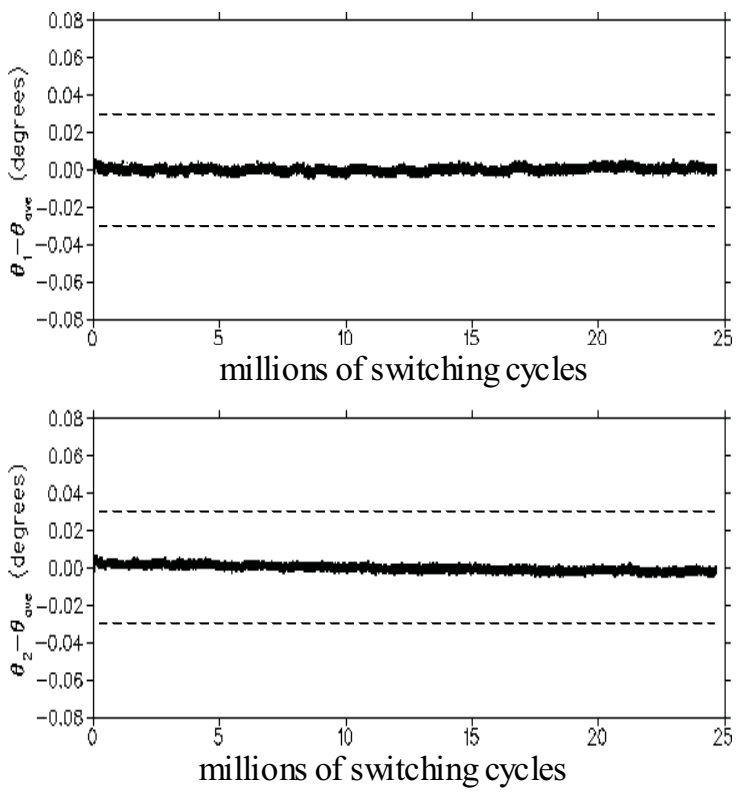

Figure 9. A plot of the change in elevation angle for two positions during a 25 million cycle wear experiment in $7 \%$ RH. The dotted lines contain the allowable change in elevation angle for $0.5 \mathrm{~dB}$ insertion loss repeatability.

A MEMS mirror with 32 positions was tested over 25 million cycles inside a dry box at 7\% RH and a temperature of $\sim 25$ Celsius. The mirror pointing angle was monitored optically with a resolution of 0.003 degrees. The mirror was first actuated around all 32 positions for 250,000 cycles, and all angles measured every 32,000 points. The data is shown in Figure 8. This test demonstrates that the MEMS mirror is capable of switching to all 32 states, and that the repeatability in angle is $\sim 0.003$ degrees, which is substantially better than 0.06 degrees required for $0.5 \mathrm{~dB}$ repeatability in insertion loss in a $32 \times 32$ optical switch. The mirror was then switched between two adjacent states for nearly 25 million cycles without interruption. The mirror pointing angle was measured optically with a resolution of 0.003 degrees. The data from the test is shown in Figure 9. 
Many of the same design features of the digital MEMS switch that minimize its vulnerability to wear also tend to minimize the probability of failure due to stiction. In comparison to other MEMS switches, the mirror structure is much larger and the ratio of kinetic energy to the surface forces from contact at the small kinematic points is much greater. The electrostatic actuation forces are also much larger than for conventional MEMS, which makes overcoming stiction easier.

We have calculated the forces that act on the digital MEMS micromirror to understand and predict the stiction of the mirror under different relative humidity $(\mathrm{RH})$ conditions. Below is a list of the forces for the micromirrors being tested.

1. Springs provide a separation force of 6 micro-Newtons

2. Electrostatic rolling separation force is 50 micro-Newtons at 100 Volts

3. Capillary adhesion force

- $\quad 52 \%$ RH (lab environment) $\sim 100$ micro-Newtons

- $7 \%$ RH (dry box) 14 micro-Newtons

Based on these results, it was expected that the capillary adhesion force would exceed both the spring separation force and the electrostatic rolling force at $52 \% \mathrm{RH}$. This matched experiments carried out on the micromirrors in lab environment. Stiction was only overcome by the rolling force when using 200300 Volts. In addition, it was expected that the electrostatic rolling force (100 Volts) would exceed the capillary adhesion force at $7 \% \mathrm{RH}$. This also matched experiments carried out on the micromirrors in a dry box environment with a measured $\mathrm{RH}$ of $7 \%$. Furthermore, there was no stiction during wear testing of the micromirror devices which underwent 25 million cycles, which indicates that there is no appreciable change in capillary adhesion forces over this many cycles.

\section{CONCLUSION}

We have demonstrated digital MEMS mirrors with up to 32 accurate mechanically prescribed positions. We have discussed the efforts taken to make these devices extremely reliable, and demonstrated mirrors with no measurable degradation after 25 million cycles. These digital MEMS mirrors enable, among other things, three dimensional digital optical switches that are smaller and lower cost than competing approaches.

\section{REFERENCES}

1. Marxer, C., et al., "Vertical Mirrors Fabriated by Deep Reactive Ion Etching for Fiber-Optic Switching Application", Journal of Microelectromechanical systems, Vol. 6, No. 3: 277-285, (1997).

2. Miller, R.A., et al., "An Electromagnetic MEMS 2x2 Fiber Optic Bypass Switch”,Transducers '97, Vol.1., (1997)

3. A. Neukermans, et al., "MEMS Technology for Optical Networking Applications", IEEE Communications Magazine, pp. 62-69, January, (2001); and references therein.

4. P. Dobbelaere, et al, "Digital MEMS for Optical Switching," IEEE Communications Magazine, vol. 40, no. 3, pp. 88-95, Mar. (2002)
5. M. Mita et al, "Multiple-height Microstructures Fabricated by ICP-RIE and Embedded Masking Layers," Trans. IEE Japan, Vol. 120-E, No. 11, 2000, pp.493-497.

6. W. Robert Ashurst, et al., "Alkene based monolayer films as anti-stiction coatings for polysilicon MEMS“, Sensors and Actuators A 91, 239-248 (2001).

7. R. Maboudian and R.T. Howe, "Critical review: Adhesion in surface micromechanical structures," J. Vac. Sci. Technol. B, vol. 15, pp.1-20, (1997); and references therein.

8. N. Tas, et al., "Stiction in surface micromachining", J. Micromech. Microeng., vol.6, pp. 385-397, (1996); and references therein. 